\title{
Papel do pensamento crítico na tomada de decisão pelo enfermeiro: revisão integrativa
}

\author{
Role of critical thinking in nurses' decision making: integrative review \\ Papel del pensamiento crítico en la toma de decisión por el enfermero: revisión \\ integradora
}

Franciane Silva Luiz ${ }^{1 *}$, Luciana Joaquina de Vasconcellos², Amanda Conrado Silva Barbosa ${ }^{3}$, Andyara do Carmo Pinto Coelho Paiva ${ }^{1}$, Kelli Borges dos Santos ${ }^{1}$, Nádia Fontoura Sanhudo ${ }^{1}$, Fábio da Costa Carbogim¹.

\section{RESUMO}

Objetivo: identificar as evidências disponíveis na literatura sobre o papel do pensamento crítico na tomada de decisão pelo enfermeiro. Métodos: Revisão integrativa, na qual o levantamento bibliográfico foi realizado nas bases de dados Medical Literature Analysis and Retrieval System Online, Cumulative Index to Nursing and Allied Health Literature e Literatura Latino-americana e do Caribe em Ciências da Saúde, no período de 26 de janeiro a 07 de fevereiro de 2018. Utilizou-se os descritores thinking, decision making e nurses. Amostra foi composta por nove artigos. Resultados: Houve aumento nas produções entre os anos de 2009 e 2014, a maioria das publicações são norte-americanas e caracterizadas por estudos de intervenção. Houve prevalência de estudos com nível de evidência III e V. Evidenciou-se três categorias de análise: Relação do pensamento crítico com a tomada de decisão de enfermeiros; Metodologias de ensino para mediar o pensamento crítico; Fatores relacionados ao desenvolvimento do pensamento crítico e à tomada de decisão. Considerações finais: $O$ pensamento crítico apresenta estreita relação com a tomada de decisão do enfermeiro. As metodologias de ensino podem mediar o desenvolvimento dessa competência. Outros fatores como a idade dos profissionais e os anos de experiência também estiveram relacionados ao pensamento crítico.

Palavras-chave: Enfermagem, Pensamento, Tomada de decisões.

\begin{abstract}
Objective: To identify the available evidence in the literature about the role of critical thinking in decision making by nurses. Methods: In integrative review, in which the bibliographic survey was carried out in the dados Medical Literature Analysis and Retrieval System Online, Cumulative Index to Nursing and Allied Health Literature and Literatura Latino-americana e do Caribe em Ciências da Saúde databases, from January 26 to February 7, 2018. The descriptors thinking, decision making and nurses. Sample was composed of nine articles. Results: There was an increase in productions between the years of 2009 and 2014, the majority of the publications are North American and characterized by intervention studies. There was a prevalence of studies with level of evidence III and V. Three categories of analysis were analyzed: Critical thinking relation with the decision-making of nurses; Teaching methodologies to mediate critical screening; Factors related to the development of critical thinking and decision making. Final considerations: Critical thinking is closely

${ }^{1}$ Universidade Federal de Juiz de Fora (UFJF), Juiz de Fora-MG. *E-mail: franciane.silva.192@gmail.com

2 Universidade Federal de Minas Gerais, Belo Horizonte-MG.

${ }^{3}$ Instituto Federal de Educação, Ciência e Tecnologia do Sudeste de Minas Gerais, São João del- Rei-MG.
\end{abstract}


related to nurses' decision making. Teaching methodologies can mediate the development of this competence. Other factors such as age of professionals and years of experience have also been related to critical thinking.

Key words: Nursing, Thinking, Decision making.

\section{RESUMEN}

Objetivo: identificar las evidencias disponibles en la literatura sobre el papel del pensamiento crítico en la toma de decisión por el enfermero. Métodos: Revisión integradora, en la cual el levantamiento bibliográfico fue realizado en las bases de datos dados Medical Literature Analysis and Retrieval System Online, Cumulative Index to Nursing and Allied Health Literature y Literatura Latino-americana e do Caribe em Ciências da Saúde, en el período del 26 de enero al 07 de febrero de 2018. Se utilizaron los descriptores thinking, decision making y nurses. La muestra fue compuesta por nueve artículos. Resultados: Hubo aumento en las producciones entre los años 2009 y 2014, la mayoría de las publicaciones son norteamericanas y caracterizadas por estudios de intervención. La prevalencia de estudios con nivel de evidencia III y V. Se evidenció tres categorías de análisis: Relación del pensamiento crítico con la toma de decisión de enfermeros; Metodologías de enseñanza para mediar el abismo crítico; Factores relacionados con el desarrollo del pensamiento crítico y la toma de decisiones. Consideraciones finales: El pensamiento crítico presenta estrecha relación con la toma de decisión del enfermero. Las metodologías de enseñanza pueden mediar el desarrollo de esta competencia. Otros factores como la edad de los profesionales y los años de experiencia también estuvieron relacionados con el pensamiento crítico.

Palabras clave: Enfermería, Pensamiento, Toma de decisiones.

\section{INTRODUÇÃO}

As premissas ideológicas que emergiram com a reforma sanitária e o Sistema Único de Saúde (SUS) brasileiro delinearam a reconfiguração da produção em saúde, a partir das necessidades da população, o rápido desenvolvimento tecnológico e os novos modos de fazer saúde. Nesse sentido, a produção de cuidado exige dos profissionais de saúde - pensamento crítico $(P C)$, reflexivo, habilidades relacionais e competências técnicas, éticas e humanas (CARBOGIM FDC, et al., 2018; CASSIANE SHB, et al., 2017).

Estas competências permeiam fortemente o cuidado em enfermagem, sendo o PC essencial ao processo de trabalho, por articular o raciocínio clínico e a tomada de decisão a partir de preceitos de qualidade e efetividade (CARBOGIM FDC, et al., 2017; DIAS JAA, et al., 2016).

Conceitualmente, o PC é estratificado em disposições, entendendo-se como a intenção e a vontade para fazer algo e por habilidades que consiste na competência em realizar determinada ação com vistas a um objetivo (CARBOGIM FDC, et al., 2016; GUZZO V e GUZZO GB, 2014).

Nesta perspectiva, compreende-se por PC, um conjunto de disposições e habilidades, que implicam na forma de se pensar sobre qualquer temática, analisá-la, julgá-la e determinar as melhores intervenções para uma tomada de decisão mais efetiva, mediante a situação e os recursos disponíveis, naquele determinado momento. Dessa forma, pensar criticamente na área da saúde e na enfermagem é fundamental para o desenvolvimento de ações seguras e livre de danos decorrentes de imperícia, negligência e imprudência (CARBOGIM FDC, et al., 2017; CARDOSO LS, et al., 2016; CASSIANE SHB, et al., 2017).

Na prática de enfermagem, destaca-se a importância do PC para julgamento clínico que conduzirá aos diagnósticos de enfermagem, as intervenções e reavaliação do plano de cuidados (OLIVEIRA LB, et al., 2016).

Nesse sentido, estudos tem demonstrado relação entre habilidades do PC com julgamentos clínicos complexos e tomada de decisão no processo de aprendizagem e no contexto da assistência à saúde (CARBOGIM FDC, et al., 2017; LUDIN SM, 2018). 
Diante o exposto, os profissionais de saúde, com ênfase no enfermeiro, devem ser capazes de tomar decisões, visando a efetividade de suas ações por meio do custo benefício entre à assistência segura e de qualidade e o dimensionamento de recursos físicos, materiais e financeiros. Assim, o PC é essencial para avaliar, sistematizar e decidir as condutas mais adequadas, baseadas em evidências científicas. Assim, além de promover uma assistência de qualidade e livre de danos, o enfermeiro possibilitará o seu reconhecimento técnico e gerencial (DUTRA HS, et al., 2016).

Ademais, há evidências que o estímulo do $P C$ no ensino de graduação tem papel importante na aquisição de conhecimento (CARBOGIM FDC, et al., 2018; WINTERS JRF, et al., 2017). Contudo, a literatura carece de estudos de revisão que sumarizem o papel do PC na tomada de decisão do enfermeiro, justificando-se a realização desse estudo. O objetivo deste estudo é realizar uma revisão integrativa para identificar as evidências disponíveis na literatura sobre o papel do PC na tomada de decisão pelo enfermeiro.

\section{MÉTODOS}

Trata-se de uma revisão integrativa para a identificação de produções científicas sobre o tema pensamento crítico na tomada de decisão pelo enfermeiro. Esse estudo seguiu as seis etapas propostas por Souza MT, et al. (2010), a saber: 1) elaboração da pergunta norteadora; 2) busca ou amostragem na literatura; 3) coleta de dados; 4) análise crítica dos estudos incluídos; 5) discussão dos resultados; 6) apresentação da revisão integrativa.

Para elaboração da pergunta norteadora, utilizou-se a estratégia do acrômio PICO, em que (P) representa Paciente ou Problema, (I) representa Intervenção, (C) representa Comparação e (O) representa Desfecho (SANTOS CMC, et al., 2007). Nesse estudo definiu-se: (P) enfermeiros, (I) pensamento crítico e (O) tomada de decisão. Destarte, a pergunta norteadora da pesquisa foi "que evidências científicas estão presentes na literatura acerca do papel do PC na tomada de decisão pelo enfermeiro?

A busca na literatura foi realizada entre 26 de janeiro e 7 de fevereiro de 2018, nas bases de dados Medical Literature Analysis and Retrieval System Online (MEDLINE via Pubmed), Cumulative Index to Nursing and Allied Health Literature (CINAHL) e Literatura Latino-americana e do Caribe em Ciências da Saúde (LILACS). O critério para escolha das bases foi a abrangência nas áreas da saúde e de enfermagem.

Realizou-se busca atemporal, utilizando-se Descritores em Ciências da Saúde (DeCs) e Medical Subject Headings Terms (MeSH) "thinking", "nurses", "decision making", intermediados pelo operador boleano "AND". Definiu-se como critérios de inclusão: todas as categorias de artigos, disponíveis na integra, com acesso livre, publicados nos idiomas português, inglês ou espanhol e que retratassem no título ou resumo os termos "thinking", "decision making" e "nurses". Dissertações e teses, notas ao editor e artigos repetidos foram excluídos do estudo.

Para a coleta de dados e, posteriormente, caracterização das produções selecionadas, utilizou-se um instrumento adaptado que contemplava os seguintes itens: título, autores, país de origem do estudo, idioma, ano de publicação, objetivos, fonte, delineamento do estudo, nível de evidência, resultados e conclusões. $O$ nível de evidência foi determinado conforme o delineamento dos estudos.

Para análise, realizou-se organização e tabulação dos dados, utilizando-se estatística descritiva. Posteriormente, procedeu-se a discussão dos resultados, com interpretação e síntese dos dados, identificando-se as implicações para a prática do enfermeiro.

\section{RESULTADOS}

A busca nas bases de dados resultou em 833 artigos relacionados ao tema proposto. Com base nos critérios de exclusão, foram considerados para avaliação, 510 estudos. Destes, realizou-se a análise dos títulos, excluindo-se 479 artigos e selecionando 31 para leitura dos resumos. Após essa etapa, 11 artigos foram excluídos, elegendo-se 20 trabalhos para a leitura na íntegra. Destes, nove foram incluídos na amostra final dessa revisão, mediante sua equivalência com o objetivo do presente estudo (Figura 1). 
Figura 1 - Fluxograma do processo de seleção dos artigos nas bases de dados.

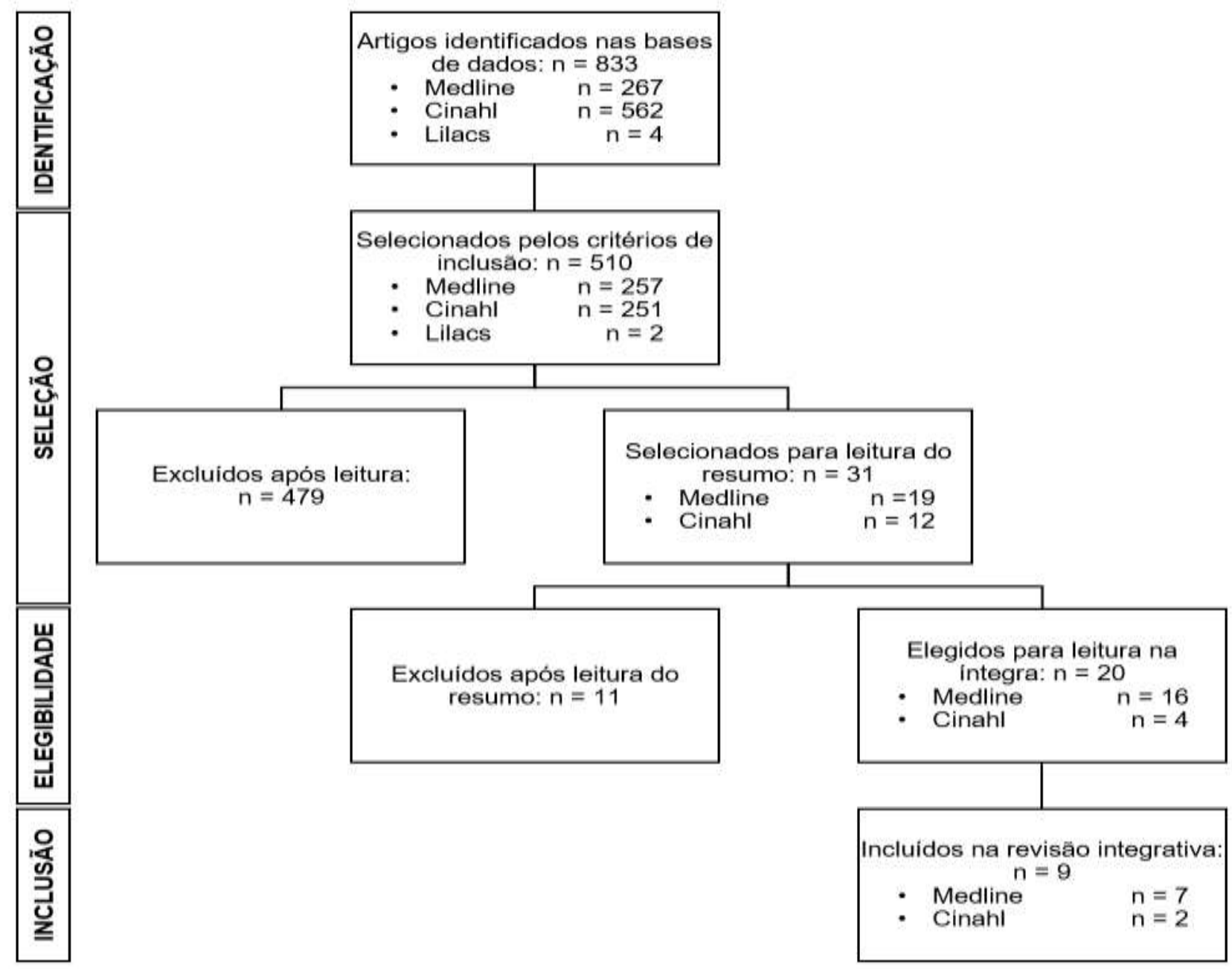

Fonte: Luiz FS, et al., 2019.

Destarte, a amostra da revisão foi constituída por nove trabalhos, sendo sete $(77,8 \%)$ dos estudos provenientes da MEDLINE e dois (22,2\%) resultantes da base de dados CINAHL, publicados entre os anos de 2003 e 2018. Do total, apenas dois (22,2\%) foram publicados nos últimos cinco anos. Entre os anos de 2004 e 2013 foram publicados seis (66,7\%) artigos e anteriormente à essa data, apenas um $(11,1 \%)$ estudo foi encontrado, sendo este do ano de 2003.

Com relação ao idioma, apenas $11,1 \%$ dos artigos foi publicado na língua portuguesa e os demais $(88,9 \%)$ na língua inglesa. A maioria das pesquisas foram realizadas nos EUA (44,5\%), seguido do Brasil (22,2\%), de Taiwan $(22,2 \%)$ e da Malásia (11,1\%).

Com relação ao delineamento dos estudos analisados, seis $(66,7 \%)$ apresentavam abordagem quantitativa, dos quais três $(50 \%)$ eram intervenções, dois $(33,3 \%)$ estudos transversais e um $(16,7 \%)$ ensaio clínico randomizado. Os demais $(33,3 \%)$ são de abordagem qualitativa.

Quanto ao nível de evidência, constatou-se que um (11,1\%) artigo apresentou nível de evidência II, dois (22,3\%) nível de evidência III e seis (66,7\%) artigos apresentam nível de evidência IV.

Os artigos elegidos foram caracterizados, no que se refere a: identificação, título em inglês e português, autores (ano de publicação), país de origem do estudo e idioma. Foi especificado, ainda, a classificação metodológica: objetivo, base de dados em que foi encontrado, delineamento do estudo e nível de evidência; e, os resultados encontrados: síntese dos resultados (Quadro 1). 
Quadro 1 - Caracterização dos artigos quanto à identificação, classificação metodológica e síntese dos resultados encontrados.

\begin{tabular}{|c|c|c|c|c|}
\hline Título & $\begin{array}{l}\text { Autores (ano)/ } \\
\text { país/ idioma }\end{array}$ & Objetivo & $\begin{array}{c}\text { Base de dados/ } \\
\text { delineamento/ nível de } \\
\text { evidência }\end{array}$ & Resultados \\
\hline $\begin{array}{l}\text { Critical thinking and } \\
\text { clinical decision } \\
\text { making in critical care } \\
\text { nursing: a pilot study }\end{array}$ & $\begin{array}{l}\text { Hicks FD, et al., } \\
(2003) \\
\text { EUA } \\
\text { Inglês }\end{array}$ & $\begin{array}{l}\text { Examinar a relação de nível } \\
\text { de educação, anos de } \\
\text { experiência de enfermagem } \\
\text { de cuidados intensivos e } \\
\text { capacidade de PC } \\
\text { (habilidades e disposições) } \\
\text { para a consistência na } \\
\text { tomada de decisão clínica. }\end{array}$ & $\begin{array}{l}\text { MEDLINE; CINAHL } \\
\text { Estudo não experimental } \\
\text { e correlacional } \\
\text { Nível IV }\end{array}$ & $\begin{array}{l}\text { Não houve associação entre o nível de educação e } \\
\text { experiência e as disposições para o PC dos } \\
\text { enfermeiros. Também não houve associação entre as } \\
\text { disposições para o PC e a coerência das decisões clíni- } \\
\text { cas. Porém, os anos de experiência clínica em } \\
\text { cuidados intensivos de enfermagem relacionaram-se } \\
\text { ao aumento da coerência na tomada de decisões. }\end{array}$ \\
\hline 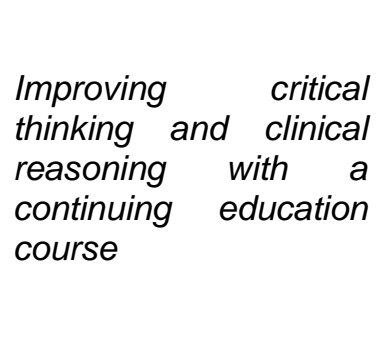 & $\begin{array}{l}\text { Cruz DM, et al., } \\
(2009) \\
\text { Brasil } \\
\text { Inglês }\end{array}$ & $\begin{array}{l}\text { Apoiar a hipótese de que a } \\
\text { participação em um curso de } \\
\text { educação continuada sobre } \\
\text { PC e raciocínio clínico } \\
\text { melhoraria a precisão dos } \\
\text { diagnósticos } \\
\text { participantes. }\end{array}$ & $\begin{array}{l}\text { CINAHL } \\
\text { Estudo } \\
\text { experimental } \\
\text { Nível III }\end{array}$ & $\begin{array}{l}\text { Encontrou-se que } 20,5 \% \text { dos participantes melhoraram } \\
\text { no estudo de caso } 1 \text { e } 38,5 \% \text { melhoraram no estudo de } \\
\text { caso } 2 \text {. Esses achados foram considerados como } \\
\text { satisfatório pelas autoras, porque entenderam que o } \\
\text { foco do curso foi o PC e o raciocínio clínico para } \\
\text { situações práticas. Destarte, o PC e raciocínio clínico } \\
\text { influenciam positivamente os escores de precisão diag- } \\
\text { nóstica dos enfermeiros }\end{array}$ \\
\hline $\begin{array}{l}\text { Critical thinking } \\
\text { competence and } \\
\text { disposition } \\
\text { of clinical nurses in a } \\
\text { medical center }\end{array}$ & $\begin{array}{l}\text { Feng RC, et al., } \\
(2010) \\
\text { Taiwan } \\
\text { Inglês }\end{array}$ & $\begin{array}{l}\text { Medir a competência e a } \\
\text { disposição do PC dos } \\
\text { enfermeiros clínicos, bem } \\
\text { como explorar os fatores } \\
\text { relacionados da competência } \\
\text { do PC. }\end{array}$ & $\begin{array}{l}\text { MEDLINE } \\
\text { Estudo } \\
\text { experimental } \\
\text { Nível III }\end{array}$ & $\begin{array}{l}\text { Encontrou-se associação da competência e a } \\
\text { disposição para o PC com o tempo de experiência } \\
\text { (mais de } 10 \text { anos) e a experiência em hospitais di- } \\
\text { ferentes. O PC também se relacionou à idade do } \\
\text { enfermeiro (média de } 32,5 \text { anos). }\end{array}$ \\
\hline
\end{tabular}




\begin{tabular}{|c|c|c|c|c|}
\hline $\begin{array}{l}\text { New Graduate Nurses' } \\
\text { Perceptions of the } \\
\text { Effects of Clinical } \\
\text { Simulation on Their } \\
\text { Critical Thinking, } \\
\text { Learning, and } \\
\text { Confidence }\end{array}$ & $\begin{array}{l}\text { Kaddoura } \\
\text { (2010) } \\
\text { EUA } \\
\text { Inglês }\end{array}$ & $\begin{array}{l}\text { Explorar as percepções de } \\
\text { enfermeiros recém-formados } \\
\text { da promoção do PC no } \\
\text { contexto da simulação clínica } \\
\text { durante o treinamento em } \\
\text { enfermagem em cuidados in- } \\
\text { tensivos. }\end{array}$ & $\begin{array}{l}\text { CINAHL } \\
\text { Estudo qualitativo } \\
\text { descritivo exploratório } \\
\text { Nível IV }\end{array}$ & $\begin{array}{l}\text { Evidenciou-se que a estratégia de ensino de simulação } \\
\text { clínica utilizada no treinamento em terapia intensiva foi } \\
\text { vital na promoção de habilidades de PC em enfermei- } \\
\text { ros recém-formados. A simulação foi descrita como um } \\
\text { processo interativo de ensino-aprendizagem, que } \\
\text { facilita o processo de aprender e pensar criticamente } \\
\text { sobre intervenções de enfermagem. }\end{array}$ \\
\hline $\begin{array}{l}\text { Critical thinking } \\
\text { reported enhancers } \\
\text { and barriers by nurses } \\
\text { in long-term care: } \\
\text { implications for staff } \\
\text { development }\end{array}$ & $\begin{array}{l}\text { Raterink G, (2011) } \\
\text { EUA } \\
\text { Inglês }\end{array}$ & $\begin{array}{l}\text { Avaliar o PC dos Enfermeiros } \\
\text { durante suas práticas, por } \\
\text { meio de instrumentos que } \\
\text { determinam os fatores no } \\
\text { ambiente de trabalho que } \\
\text { melhoram ou servem como } \\
\text { barreiras para a imple- } \\
\text { mentação do PC. }\end{array}$ & $\begin{array}{l}\text { MEDLINE } \\
\text { Estudo } \\
\text { naturalista } \\
\text { Nível IV }\end{array}$ & $\begin{array}{l}\text { Os enfermeiros determinaram como aspectos } \\
\text { promotores para a implementação do PC no ambiente } \\
\text { de trabalho: cooperação entre colaboradores, abertura } \\
\text { e respeito a diferentes concepções (sobre PC), cultura } \\
\text { e estrutura organizacional. } \\
\text { Barreiras identificadas para a implementação do PC: } \\
\text { tempo limitado, falta de pessoal, o medo da perda de } \\
\text { emprego, o estresse, os cuidados tecnicamente } \\
\text { focados, a documentação atrasada e as atividades que } \\
\text { aumentam as demandas no tempo e energia. }\end{array}$ \\
\hline $\begin{array}{l}\text { Case studies } \\
\text { combined with or } \\
\text { without concept maps } \\
\text { improve critical } \\
\text { thinking in hospital- } \\
\text { based nurses: a } \\
\text { randomized-controlled } \\
\text { trial }\end{array}$ & $\begin{array}{l}\text { Huang YC, et al., } \\
(2012) \\
\text { Taiwan } \\
\text { Inglês }\end{array}$ & $\begin{array}{l}\text { Avaliar os efeitos de um } \\
\text { programa de estudos de } \\
\text { caso, isolados ou combi- } \\
\text { nados com mapas con- } \\
\text { ceituais, na melhoria do PC } \\
\text { em enfermeiros assistenciais. }\end{array}$ & $\begin{array}{l}\text { MEDLINE; CINAHL } \\
\text { Ensaio clínico } \\
\text { randomizado } \\
\text { Nível II }\end{array}$ & $\begin{array}{l}\text { Após o programa, houve diferenças significativas entre } \\
\text { os grupos experimental e controle nas habilidades de } \\
\text { PC de análise, avaliação, inferência, dedução e } \\
\text { indução. O grupo experimental demonstrou melhoria } \\
\text { significativa na análise, avaliação e dedução, no pré e } \\
\text { pós-teste. Já o grupo controle demonstrou diferença } \\
\text { negativa na análise e dedução, no pré e pós-teste. En- } \\
\text { controu-se também diferença significativa na dispo- } \\
\text { sição específica de abertura mental, tendo o grupo } \\
\text { experimental demonstrado melhorias do pré-teste para } \\
\text { o pós-teste no que se refere à mente aberta, analítica, } \\
\text { sistematização, autoconfiança e curiosidade. O grupo } \\
\text { controle não demonstrou diferença entre o pré e o pós- } \\
\text { teste. Constatou-se que os estudos de caso com- } \\
\text { binados com mapas conceituais foram mais efetivos do } \\
\text { que os estudos de caso sozinhos para melhorar as } \\
\text { habilidades de PC. As habilidades de PC melhoradas } \\
\text { incluíram avaliar problemas clínicos, planejar } \\
\text { intervenções de enfermagem e resolver problemas. }\end{array}$ \\
\hline
\end{tabular}

REAS/EJCH | Vol.Sup.n.38 | e1763 | DOI: https://doi.org/10.25248/reas.e1763.2020 Página 6 de 11 


\begin{tabular}{|c|c|c|c|c|}
\hline $\begin{array}{l}\text { A cross-sectional study } \\
\text { examining } \\
\text { factors related to } \\
\text { critical } \\
\text { thinking in nursing }\end{array}$ & $\begin{array}{l}\text { Lang GM, et al., } \\
(2013) \\
\text { EUA } \\
\text { Inglês }\end{array}$ & $\begin{array}{l}\text { Examinar habilidades de PC } \\
\text { entre enfermeiras que } \\
\text { trabalham em um hospital } \\
\text { militar. }\end{array}$ & $\begin{array}{l}\text { MEDLINE } \\
\text { Estudo quantitativo } \\
\text { transversal } \\
\text { Nível IV }\end{array}$ & $\begin{array}{l}\text { Não encontrou associação significativa entre } \\
\text { habilidades de PC, idade e anos de experiência. No } \\
\text { entanto, as diferenças foram identificadas entre os } \\
\text { grupos raciais/étnicos, tendo os caucasianos e hispâ- } \\
\text { nicos apresentado maiores habilidade de PC em } \\
\text { relação aos afro-americanos. }\end{array}$ \\
\hline $\begin{array}{l}\text { Structural elements of } \\
\text { critical thinking of } \\
\text { nurses in emergency } \\
\text { care } \\
\text { Elementos estruturais } \\
\text { do pensamento crítico } \\
\text { de enfermeiros } \\
\text { atuantes em } \\
\text { emergências }\end{array}$ & $\begin{array}{l}\text { Crossetti MGO, et } \\
\text { al., (2014) } \\
\text { Brasil } \\
\text { Português }\end{array}$ & $\begin{array}{l}\text { Analisar os elementos } \\
\text { estruturais do PC do en- } \\
\text { fermeiro no processo de } \\
\text { tomada de decisão clínica. }\end{array}$ & $\begin{array}{l}\text { MEDLINE; LILACS } \\
\text { Estudo } \quad \text { qualitativo } \\
\text { exploratório } \\
\text { Nível IV }\end{array}$ & $\begin{array}{l}\text { Os enfermeiros do estudo identificaram e definiram os } \\
\text { elementos estruturais constituintes do PC que, na } \\
\text { avaliação deles constituíam o PC e conduziam à } \\
\text { tomada de decisão clínica. Os elementos foram } \\
\text { conhecimento técnico-científico e experiência clínica, } \\
\text { os quais representam a relação entre os fundamentos } \\
\text { teóricos e práticos para a tomada de decisão; raciocínio } \\
\text { clínico, julgamento clínico, intuição, predição, elemen- } \\
\text { tos que representam o processo de pensamento e } \\
\text { tomada de decisão clínica; }\end{array}$ \\
\hline $\begin{array}{l}\text { Does good critical } \\
\text { thinking equal effective } \\
\text { decision-making } \\
\text { among critical care } \\
\text { nurses? } A \text { cross- } \\
\text { sectional survey }\end{array}$ & $\begin{array}{l}\text { Ludin SM (2018) } \\
\text { Malásia } \\
\text { Inglês }\end{array}$ & $\begin{array}{l}\text { Entender se a disposição do } \\
\text { PC dos enfermeiros de } \\
\text { cuidados críticos afeta suas } \\
\text { habilidades de decisão } \\
\text { clínica. }\end{array}$ & $\begin{array}{l}\text { Estudo } \quad \text { quantitativo } \\
\text { transversal } \\
\text { Nível IV }\end{array}$ & $\begin{array}{l}\text { Encontrou-se relação forte e positiva entre o PC dos } \\
\text { enfermeiros de cuidados intensivos e a sua tomada de } \\
\text { decisão clínica. Os enfermeiros de cuidados críticos } \\
\text { apresentaram níveis elevados de PC e de tomada de } \\
\text { decisão clínica. A idade (média de } 33,85 \text { anos) e a } \\
\text { experiência de trabalho (média de } 8,51 \text { anos) } \\
\text { impactaram significativamente para a tomada de } \\
\text { decisão clínica. }\end{array}$ \\
\hline
\end{tabular}

Fonte: Luiz FS, et al., 2019. 


\section{DISCUSSÃO}

Os artigos incluídos nesta revisão contribuíram de forma significativa para o desenvolvimento da temática, apesar de apenas um deles possuir nível de evidência I ou II. Contudo, independentemente do nível de evidência, todos os estudos incluídos nessa revisão contribuíram de forma significativa para descrever a importância do PC na tomada de decisão pelo enfermeiro. Evidenciou-se um aumento na produção científica entre os anos de 2009 e 2014. Após a leitura e sumarização dos dados emergiram três categorias, a saber: Relação do PC com a tomada de decisão de enfermeiros; Metodologias de ensino para mediar o PC; Fatores relacionados ao desenvolvimento do $\mathrm{PC}$ e à tomada de decisão.

\section{Relação do PC com a tomada de decisão de enfermeiros}

O PC é uma competência essencial para enfermeiros com o domínio de habilidades intelectuais, interpessoais e técnicas e, possibilita o julgamento conciso e a tomada de decisão efetiva (MENEZES SSC, et al., 2015). Dos nove estudos incluídos nessa revisão, dois corroboram com essa afirmação (CROSSETTI MGO, et al., 2014; LUDIN SM, 2018). Diversamente, uma das amostras descreve que não há relação entre o PC e a tomada de decisão (HICKS FD, et al., 2003).

Um estudo transversal e realizado na Malásia (LUDIN SM, 2018),encontrou forte relação entre o PC de enfermeiros de cuidados críticos e a sua tomada de decisão clínica, identificando que esses profissionais apresentaram níveis elevados de PC e de tomada de decisão clínica. Para a realização desse estudo, os autores utilizaram questionários autoaplicáveis, constituídos por questões demográficas, pelo Short Form Malay and English version of the Critical Thinking Disposition Inventory (SF-CTDI-CV) e Clinical decisionmaking in nursing scale (CDMNS). O SF-CTDI-CV trata-se da versão reduzida do Critical Thinking Disposition Inventory, validado em malaio e em inglês e destinada a avaliar o pensamento crítico de enfermeiros na prática. Para mensuração da tomada de decisão clínica em enfermagem os autores utilizaram a CDMNS. Os valores do alfa de Cronbach dessas escalas foram, respectivamente, 0,74 e 0,80.

Do mesmo modo, reforça um estudo qualitativo exploratório, realizado no Brasil (CROSSETTI MGO, et al., 2014), o qual demonstrou, na perspectiva de enfermeiros assistenciais, os elementos estruturais que constituem o PC e que asseguram a tomada de decisão clínica. Conforme os participantes, esses elementos definem a relação entre o conhecimento teórico e prático para a tomada de decisão (conhecimento técnicocientífico e experiência clínica), o processo de pensamento para a tomada de decisão (raciocínio clínico, julgamento clínico, intuição, predição) e as bases para o julgamento clínico (avaliação do paciente, ética, conhecimento do paciente, conhecimento cultural, aplicação de padrões de normalidade e ética) (CROSSETTI MGO, et al., 2014).

Contrariamente, um estudo de delineamento não experimental e correlacional (HICKS FD, et al., 2003), utilizando o Decision Analytic Questionnaire (DAQ), o California Critical Thinking Disposition Inventory (CCTDI) e o California Critical Thinking Skills Test (CCTST) identificou que não há relação entre a tomada de decisão pelos enfermeiros intensivistas e o PC. O DAQ, um instrumento modificado pelos pesquisadores, foi usado para avaliar a consistência da tomada de decisão por enfermeiros de cuidados críticos. Para avaliar a disposição para o PC foi utilizado o CCTDI e para medir as habilidades para o PC, o CCTST. No que se refere às propriedades psicométricas destes instrumentos, cabe destacar que o DAQ apresentou valores aceitáveis para o teste de confiabilidade, porém testes para avaliação da consistência interna do instrumento não foram realizados. Já a CCTST apresentou valores de consistência interna inaceitáveis durante o teste preliminar e foi utilizada pois os autores relacionaram esse fato ao cansaço dos participantes, que preencheram os instrumentos (CCTST e DAQ) sem intervalo entre eles (HICKS FD, et al., 2003).

Infere-se que a divergência entre os resultados desses estudos, possivelmente, se deve aos diferentes métodos e instrumentos utilizados para realização da pesquisa. Ademais, evidencia-se que as escalas utilizadas por Ludin SM (2018), a SF-CTDI-CV e a CDMNS, apresentaram valores de confiabilidade aceitáveis (SOUZA AC, et al., 2017) e que os instrumentos, DAQ e CCTST, utilizados no estudo realizado por Hicks FD, et al. (2003) tiveram as propriedades psicométricas questionadas pelos próprios autores, o que pode ter influenciado nos resultados encontrados. 
Isto posto, destaca-se a correspondência entre os estudos realizados por Crosseti MGO, et al. (2014) e Ludin SM (2018) que atestaram, respectivamente, de forma quantitativa e qualitativa a relação entre o PC a tomada de decisão segura e efetiva, fundamentais para o cuidado sistematizado e de qualidade.

\section{Metodologias de ensino para mediar o PC}

O PC é uma competência que pode ser estimulada, ensinada e aprendida e, no processo de ensino, pode ser abordado de forma explícita quando envolvido de maneira direta, ou implícita, quando abordado indiretamente. Independente da técnica pedagógica elegida, a metodologia de ensino deve ser capaz de mobilizar o PC (CARBOGIM FDC, et al., 2017; HICKS FD, et al., 2003).

Nesta perspectiva, três estudos da amostra elegida para esta revisão destacam-se por abordarem metodologias de ensino distintas, que mediam o desenvolvimento do PC (CRUZ DM, et al. 2009; HUANG L, et al., 2015; KADDOURA MA, 2010), impactando diretamente na tomada de decisão clínica. As metodologias identificadas foram curso de educação continuada de 16 horas sobre o PC e raciocínio clínico (CRUZ DM, et al., 2009), simulação clínica (KADDOURA MA, 2010) e estudos de casos combinados com mapas conceituais (HUANG L, et al., 2015).

A aplicação de um curso de educação continuada como metodologia para mediar o PC e, por consequente, a capacidade de julgamento das condições clínicas do paciente foi descrito em um estudo do tipo intervenção realizado no Brasil. Nesse curso, os autores utilizaram estudos de caso escritos com a finalidade de se discutir sobre PC e raciocínio clínico e, constataram que essa abordagem influenciou positivamente os escores de precisão diagnóstica dos enfermeiros (CRUZ DM, et al., 2009).

Estudo realizado por Campos KFC, et al. (2017) corrobora ao reconhecer que a educação permanente, quando envolve discussões coletivas e reflexivas, bem como o uso de metodologias de ensino não convencionais e a problematização da realidade, permite que o processo de ensino e aprendizagem ocorra de forma favorável, estimulando-se práticas de ação, reflexão e ação durante o cuidado.

Ademais, Puggina CC, et al. (2015), contribuem com esse achado ao exporem que o PC pode ser estimulado pela educação permanente, a qual possibilita também o crescimento pessoal e coletivo da equipe de trabalho, por meio de um aprendizado ativo e relacionado ao contexto do serviço.

Apesar de evidenciarem a importância da educação continuada para o desenvolvimento do PC, estes estudos demonstraram dificuldades relacionadas à implementação da educação permanente, as quais exigem o envolvimento dos trabalhadores, a compreensão conceitual da expressão "educação permanente" e a alta rotatividade dos profissionais, sendo preciso investimentos nessa área (CAMPOS KFC, et al., 2017; PUGGINA CC, et al., 2015). No que se refere a simulação clínica, essa estratégia para mediar o PC foi apontada em um estudo descritivo, realizado nos EUA (KADDOURA MA, 2010).

A simulação clínica como um processo interativo de ensino-aprendizagem, facilita o processo de aprender e pensar criticamente sobre intervenções de enfermagem e, demonstra que quando utilizada no treinamento em terapia intensiva é essencial na promoção de habilidades de PC em enfermeiros recém-formados. Outro estudo de abordagem qualitativa aponta que a simulação clínica viabiliza o desenvolvimento de habilidades e de autorreflexão frente as oportunidades de aprendizagem (TEIXEIRA CRS, et al., 2015).

Além dessas metodologias, um ensaio clínico randomizado, realizado na China, constatou que os estudos de caso combinados com mapas conceituais foram mais efetivos para melhorar as habilidades de PC em enfermeiros assistenciais, quando comparados com os estudos de caso isolados. Além disso, o mesmo estudo identificou que as habilidades de PC melhoradas incluíam avaliar problemas clínicos, planejar intervenções de enfermagem e resolver problemas (HUANG L, et al., 2015), o que viabiliza um cuidado sistematizado, holístico e seguro.

Souza GM, et al. (2015) também constataram que o mapa conceitual permite o desenvolvimento do PC, o que reflete na determinação de ações e na resolução de problemas de forma efetiva, assim como em uma assistência de enfermagem holística. 
Assim, apesar da heterogeneidade dos trabalhos encontrados, no que se refere ao país de desenvolvimento da pesquisa e ao método de ensino utilizado, evidenciou-se que as metodologias de ensino mediam de forma apropriada o desenvolvimento do PC em enfermeiros, o que reflete na tomada de decisão e efetividade do cuidado.

\section{Fatores relacionados ao desenvolvimento do PC e à tomada de decisão}

A idade do profissional e o tempo de experiência clínica em enfermagem são fatores relacionados ao desenvolvimento do PC e à tomada de decisão clínica (FENG RC, et al., 2010; HICKS FD, et al., 2003; LUDIN SM, 2018). Ludin SM (2018) relacionou a média de 8,51 anos de experiência de trabalho e a idade média 33,85 anos à tomada de decisão clínica mais efetiva. Outro estudo reforça esta associação, no entanto, considera o tempo de experiência superior a 10 anos e a idade do indivíduo, com média, de 32,5 anos (FENG RC, et al., 2010). Em contrapartida, estudo realizado por Lang GM, et al. (2013) apontou que não há associação significativa entre o PC e idade e anos de experiência. Porém, os autores encontraram relação entre o PC e os grupos raciais / étnicos, identificando-se que os caucasianos e hispânicos apresentam maiores habilidades de $\mathrm{PC}$ em relação aos afro-americanos.

Hicks FD, et al. (2003) identificaram ainda que não há relação entre o nível de educação e as disposições para o PC dos enfermeiros, permitindo refletir sobre a necessidade de (re)pensar o processo de ensino e aprendizagem no que se refere à formação de enfermeiros com disposições e habilidades para o PC. Isto posto, compreende-se que quanto maior o nível de formação, maior a capacidade de pensar criticamente.

Destaca-se ainda um estudo desenvolvido nos Estados Unidos que identificou, na perspectiva de enfermeiros, fatores favoráveis e desfavoráveis para o uso do PC durante a prática. No que se refere aos fatores favoráveis incluiu-se o incentivo à colaboração, a abertura para o PC e para os diferentes pontos de vista, a cultura e a estrutura organizacional. Em contrapartida, elementos como tempo limitado, escassez de pessoal, receio em perder o emprego, estresse, cuidados tecnicamente focados, documentação atrasada e as atividades que aumentam as demandas no tempo e energia foram eleitos como fatores desfavoráveis para a utilização do PC (RATERINK G, 2011). Entende-se assim que as habilidades para o PC podem ser estimuladas no ambiente de trabalho visando-se um julgamento clínico adequado e uma consequente tomada de decisão efetiva. Conhecer os fatores que interferem negativamente no PC contribui para repensar a prática assistencial e gerar reflexões sobre os desafios e possibilidades para superar os fatores limitantes.

\section{CONSIDERAÇÕES FINAIS}

As evidências encontradas nos estudos apontam que o PC é uma competência fundamental para a tomada de decisão efetiva pelo enfermeiro, estando estreitamente relacionada à assistência segura e de qualidade. Do mesmo modo, por estar intrinsecamente relacionado com a assistência à saúde, destaca-se que o PC pode ser desenvolvido por meio de metodologias de ensino e a sua implementação durante a prática está associada com fatores facilitadores e dificultadores presentes no ambiente de trabalho. Nesse sentido, o presente estudo faz refletir sobre a necessidade de se (re)pensar os processos formativos e pós formativos no que tange à necessidade de pensadores críticos. Ressalta-se que as limitações para a realização desta pesquisa estiveram associadas à escassez de estudos sobre o papel do PC durante a prática dos enfermeiros, associado a poucas produções com alto nível de evidência. Destarte, em busca de elucidar essas indagações, faz-se necessário produções científicas envolvendo o papel do PC na tomada de decisão pelo enfermeiro, bem como sobre as formas de mediar o desenvolvimento dessa competência.

\section{REFERÊNCIAS}

1. CAMPOS KFC, et al. Educação permanente nos serviços de saúde. Escola Anna Nery Revista de Enfermagem, 2017; ;21: e20160317. doi:10.1590/2177-9465-EAN-2016-0317.

2. CARBOGIM FDC, et al. Critical thinking: concept analysis from the perspective of Rodger's evolutionary method of concept analysis. Revista Latino-Americana de Enfermagem, 2016; 24: e2785. doi:10.1590/1518-8345.1191.2785. 
3. CARBOGIM FDC, et al. Educational intervention to improve critical thinking for undergraduate nursing students: A randomized clinical trial. Nurse Education in Practice, 2018; 33: 121-126. doi: 10.1016/j.nepr.2018.10.001.

4. CARBOGIM FDC, et al. Teaching critical thinking skills through problem-based learning. Texto \& Contexto Enfermagem, 2017; 26. doi:10.1590/0104-07072017001180017.

5. CARDOSO LS, et al. O pensar da enfermagem em serviço de urgência e emergência intra-hospitalar. Revista de Enfermagem UFPE on line, 2016; 10: 4524-4531. doi:10.5205/1981-8963-v10i12a11519p4524-4531-2016.

6. CASSIANE SHB, et al. A situação da educação em enfermagem na América Latina e no Caribe rumo à saúde universal. Revista Latino-Americana de Enfermagem, 2017; 25: e2913. doi:10.1590/1518-8345.2232.2913.

7. CROSSETTI MGO, et al. Elementos estruturais do pensamento crítico de enfermeiros atuantes em emergências. Revista Gaúcha de Enfermagem, 2014; 35: 55-60. doi:10.1590/1983-1447.2014.03.45947.

8. CRUZ DM, et al. Improving critical thinking and clinical reasoning with a continuing education course. Journal of Continuing Education in Nursing. 2009; 40: 121-127. doi:10.3928/00220124-20090301-05.

9. DIAS JAA, et al. Ciência, enfermagem e pensamento crítico - reflexões epistemológicas. Revista de Enfermagem UFPE on line, 2016; 10: 3669-3675. doi:10.5205/reuol.9681-89824-1-ED.1004sup201619.

10. DUTRA HS, et al. Utilização do processo de enfermagem em unidade de terapia intensiva: revisão integrativa da literatura. HU Revista, 2016; 42: 245-252.

11. FENG RC, et al. Critical thinking competence and disposition of clinical nurses in a medical center. The Journal of Nursing Research, 2010; 18: 77-87. doi:10.1097/JNR.0b013e3181dda6f6.

12. GUZZO V, GUZZO GB. O pensamento crítico como ferramenta de defesa intelectual. Conjectura: Filosofia e Educação, 2014; 20: 64-76.

13. HICKS FD, et al. Critical thinking and clinical decision making in critical care nursing: a pilot study. Heart \& Lung: The Journal of Critical Care, 2003; 32: 169-180. doi:10.1016/S0147-9563(03)00038-4.

14. HUANG L, et al. Exploring the association between parental rearing styles and medical students' critical thinking disposition in China. BMC Medical Education, 2015; 15: 88. doi: 10.1186/s12909-015-0367-5.

15. HUANG YC, et al. Case studies combined with or without concept maps improve critical thinking in hospital-based nurses: a randomized-controlled trial. International Journal of Nursing Studies, 2012; 49: 747-754. doi: 10.1016/j.ijnurstu.2012.01.008.

16. KADDOURA MA. New graduate nurses' perceptions of the effects of clinical simulation on their critical thinking, learning, and confidence. Journal of Continuing Education in Nursing, 2010; 41: 506-516. doi:10.3928/0022012420100701-02.

17. LANG GM, et al. A cross-sectional study examining factors related to critical thinking in nursing. Journal for Nurses in Professional Development, 2013; 29: 8-15. doi:10.1097/NND.0b013e31827d08c8.

18. LUDIN SM. Does good critical thinking equal effective decision-making among critical care nurses? A cross-sectional survey. Intensive \& Critical Care Nursing, 2018; 44: 1-10. doi: 10.1016/j.iccn.2017.06.002.

19. MENDES KDS, et al. Revisão integrativa: método de pesquisa para a incorporação de evidências na saúde e na enfermagem. Texto \& Contexto Enfermagem, 2008; 17: 758-764. doi:10.1590/S0104-07072008000400018.

20. MENEZES SSC, et al. Raciocínio clínico no ensino de graduação em enfermagem: revisão de escopo. Revista da Escola de Enfermagem da USP, 2015; 49: 1032-1039. doi:10.1590/S0080-623420150000600021.

21. OLIVEIRA LB, et al. Efetividade das estratégias de ensino no desenvolvimento do pensamento crítico de graduandos de Enfermagem: uma metanálise. Revista da Escola de Enfermagem da USP, 2016; 50: 355-364. doi:10.1590/S0080623420160000200023.

22. PUGGINA CC, et al. Educação permanente em saúde: instrumento de transformação do trabalho de enfermeiros. Espaço para a Saúde, 2015; 16: 87-97. doi:10.22421/1517- 7130.2015v16n4p87.

23. RATERINK G. Critical thinking: Reported enhancers and barriers by nurses in long-term care: implications for staff development. Journal for nurses in staff development, 2011; 27: 136-142. doi:10.1097/NND.0b013e318217b3f3.

24. SANTOS CMC, et al. A estratégia PICO para a construção da pergunta de pesquisa e busca de evidências. Revista Latino-Americana de Enfermagem, 2007; 15: 508-511. doi:10.1590/S0104-11692007000300023.

25. SOUZA AC, et al. Propriedades psicométricas na avaliação de instrumentos: avaliação da confiabilidade e da validade. Epidemiologia e Serviços de Saúde, 2017; 26: 649-659. doi:10.5123/s1679-49742017000300022.

26. SOUZA GM, et al. Construção e utilização de um mapa conceitual como método de ensino-aprendizagem: relato de experiência. Rede de Cuidados em Saúde, 2015; 9: 1-4.

27. TEIXEIRA CRS, et al. Avaliação dos estudantes de enfermagem sobre a aprendizagem com a simulação clínica. Revista Brasileira de Enfermagem, 2015; 68: 311-319. doi:10.1590/0034-7167.2015680218i.

28. WINTERS JRF, et al. Formação dialógica e participativa na enfermagem: contribuição ao desenvolvimento do pensamento crítico-reflexivo e criativo de acadêmicos. Revista Mineira de Enfermagem, 2017; 21 : 1-8. doi:10.5935/1415-2762.20170077. 